\title{
The Rotation of the Solar Radiative zone
}

\author{
S. Turck-Chieze \\ IRFU/CEA CE Saclay, 91101 Gif sur Yvette cedex, France \\ email: cturck@cea.fr
}

\begin{abstract}
Dynamical processes are progressively introduced in stellar evolution. In this framework, the Sun is a very specific case where both models and observations have been developed in parallel during the last decade in order to progress on our present insight of solar like stars. In this poster I show the recent progress done on both sides for the rotation of the radiative zone. The present knowledge of the solar rotation profile comes from the detection of acoustic and gravity modes with the instruments GOLF and MDI aboard SoHO. In parallel we study the sensitivity of the theoretical rotation profiles obtained with the CESAM code using different rotation history in the premainsequence.
\end{abstract}

Keywords. Sun: rotation, sun: general

\section{Introduction}

Dynamical processes are present in stars all across the Hertzsprung-Russell diagram. They are clearly visible in young stars, these stars are generally rapid rotators which then decelerate after their dissociation from their initial disk. Rotation plays also a major role in massive stars and more specifically in the final stages of evolution. They are generally strong rotators with strong stellar winds.

In fact, we need to introduce the internal dynamical processes (rotation, magnetic field and gravity waves) in the stellar structure equations to describe properly the different stages of evolution. This is a hard work because it supposes to replace the four structural equations by 16 complex equations, but it is necessary because the $3 \mathrm{D}$ simulations cannot yet be used to follow the evolution of stars and consequently to confront results to asteroseismic observations. In this poster we limit our ambition to the first step, which consists to compare the present observed rotation profile to the one obtained in introducing the transport of momentum due to the solar rotation.

\section{The internal observed rotation profile}

After more than 10 years of SoHO observation, we have got very important constraints on the rotation profile of the radiative zone thanks to the GOLF and MDI instruments. The determination of the splittings of a large number of acoustic modes, in particular those which are not influenced by the superficial magnetic field, has definitively established that the part of the radiative zone which is not influenced by the nuclear core, that means the region between 0.25 to $0.68 \mathrm{R}_{\odot}$ is really flat with invisible latitudinal differential variation, in great contrast with the latitudinal differential profile of the convective zone. Below $0.25 \mathrm{R}_{\odot}$, only gravity modes may inform on the rotation profile. These modes begin to be observed by the GOLF instrument (Turck-Chieze et al., 2004, Garcia et al., 2007). Two solutions have been extracted from the pattern attributed to an $\ell=2, \mathrm{n}=2$ mode depending on the number of the observed components : a slightly reduced rotation rate or an increase by about a factor 3 in the central region (Mathur et al. 2008). Indeed the solution of a rapid rotating core is compatible with the two kinds 

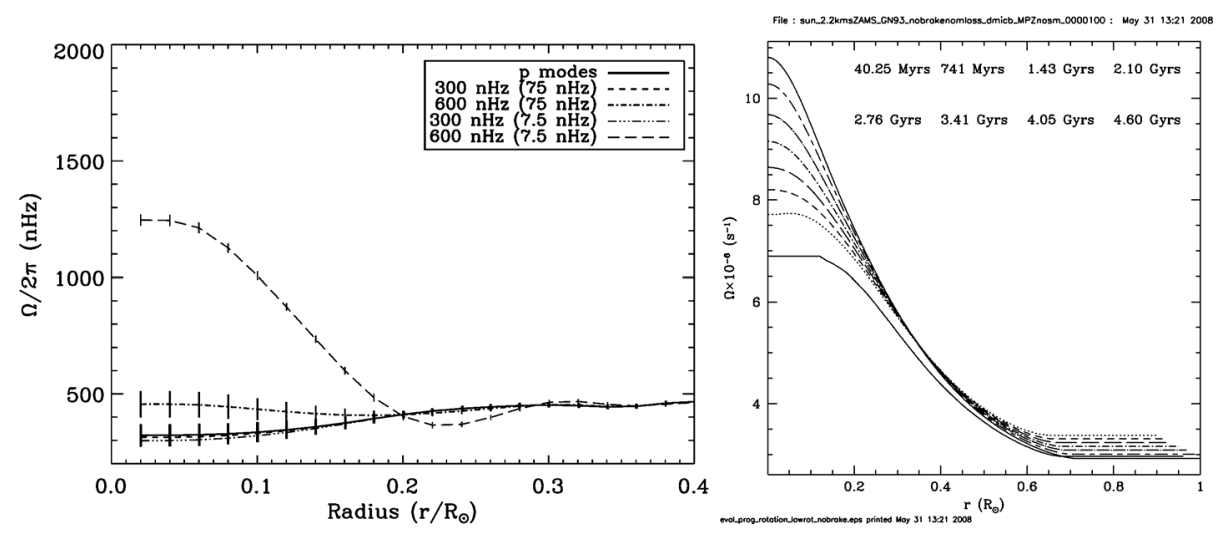

Figure 1. a) Solar observed radiative rotation profile extracted from gravity and acoustic modes using a gravity mode candidate attributed to an $\ell=2, \mathrm{n}=3$ for two values of splittings (300 and $600 \mathrm{nHz}$ ). The values $75 \mathrm{nHz}$ and $7.5 \mathrm{nHz}$ correspond respectively to a possible asymmetry of the components attributed to central magnetic field and to a typical duration of observation. From Mathur (2008). b) Evolution of the theoretical rotation profile supposing initially a flat rotation profile. From Palacios, Nghiem and Turck-Chieze (2008).

of detection (search of individual mode at high frequency, asymptotic behaviour at low frequency). It supposes also that the solar core rotation axis is different from the one of the rest of the radiative zone. This exciting possibility needs to be confirmed with an improved instrument like the GOLF-NG concept. A prototype of this instrument presently observes in Tenerife (Turck-Chieze et al. 2006 Adv Spa. Res, 2008 Astron. Notes).

\section{The internal theoretical rotation profile}

We have computed new solar models from the premainsequence including the transport of angular momentum and chemicals by rotating induced processes (shear turbulence and meridional circulation). We deduce from them the sound speed, density and rotation profiles for a direct comparison with the helioseismic observables. We demonstrate the role of the advection term in following three solar evolutions which differ by their initial conditions: low and high $(20 \mathrm{~km} / \mathrm{s}$ and $50 \mathrm{~km} / \mathrm{s})$ rotation profiles. They all lead to a differential radial rotation profile in the radiative zone but with significantly different contrast. The observed internal rotation cannot be reproduced in taking into account only these hydrodynamical processes (Fig. 1) as previously mentioned, but we demonstrate the important impact of the initial rotation for a further estimate of the role of the deep magnetic field or of the gravity waves on the transport of momentum or chemicals. These models which are physically more representative of the real Sun than the solar standard or the seismic model slightly degradate the agreement with all the observables (neutrinos and seismology) if one supposes an initial high rotation. These results demonstrate the complexity of the modelling of the solar radiative zone. They call for more theoretical efforts on the dynamical processes in radiative zones.

\section{References}

Garcia, R. A., Turck-Chieze, S., Jimenez-Reyes, S. J. et al. 2007, Science, 316, 1537

Mathur, S., Eff-Darwich, A., Garcia, R. \& Turck-Chieze, S. 2008, A\&̊A,484, 517

Palacios, Nghiem \& Turck-Chieze 2008, ApJ submitted

Turck-Chieze, S., Garca, R. A., Couvidat, S. et al. 2004, ApJ 604, 455; erratum ApJ, 604, 455 